\title{
Randomized clinical trial to evaluate ferric carboxymaltose and iron sucrose for treatment of postpartum anaemia in a tertiary hospital
}

\author{
Sheela H. S.*, Ashwini Neelakanthi, Nikitha C. P.
}

Department of Obstetrics and Gynecology, Vydehi Institute of Medical Sciences and Research Centre, Bengaluru, Karnataka, India

Received: 13 September 2018

Accepted: 05 October 2018

*Correspondence:

Dr. Sheela H. S.,

E-mail: drsheelajp@gmail.com

Copyright: (c) the author(s), publisher and licensee Medip Academy. This is an open-access article distributed under the terms of the Creative Commons Attribution Non-Commercial License, which permits unrestricted non-commercial use, distribution, and reproduction in any medium, provided the original work is properly cited.

\begin{abstract}
Background: Ferric carboxymaltose and iron sucrose are two iron carbohydrate complexes optimized for iron delivery and extensively used in postpartum anaemia. This study compares the efficacy and safety of ferric carboxymaltose with that of iron sucrose in patients with postpartum anaemia.

Methods: One hundred women diagnosed with postpartum anaemia were randomized prospectively in a $1: 1$ ratio to receive either ferric carboxymaltose or iron sucrose. Fifty patients received intravenous iron carboxymaltose depending on the iron deficit at rate of $1000 \mathrm{mg} / \mathrm{week}$ and fifty patients received intravenous iron sucrose at a rate of $200 \mathrm{mg} /$ day on alternate days till the calculated dose is given.

Results: Fifty patients were enrolled in each treatment group. Both groups showed increases in mean haemoglobin from baseline at 2 weeks and 6 weeks. The increase in haemoglobin was significantly higher in ferric carboxymaltose group compared to iron sucrose at both week $2(2.64 \pm 0.91$ versus $2.17 \pm 0.76 ; \mathrm{p}=0.010)$ and week 6 timepoints (4.65 \pm 1.17 versus $3.96 \pm 1.06 ; \mathrm{p}=0.005)$. The proportion of patients achieving target haemoglobin of $12 \mathrm{gm} / \mathrm{dl}$ was significantly higher in ferric carboxymaltose group compared to iron sucrose at week 6 (77.3\% versus 50.0\%; $\mathrm{p}=0.013)$. The incidence of adverse events was similar across treatment groups and no specific safety concerns were observed.

Conclusions: Both ferric carboxymaltose and iron sucrose caused increase in baseline haemoglobin. Ferric carboxymaltose had significantly higher increases in haemoglobin compared to iron sucrose at both follow up timepoints (week 2 and week 6). It was more likely to achieve target haemoglobin with ferric carboxymaltose within 6 weeks compared to iron sucrose. Ferric carboxymaltose may be considered in women with postpartum anaemia for faster rise in haemoglobin while requiring fewer injections compared to iron sucrose.
\end{abstract}

Keywords: Ferric carboxymaltose, Iron sucrose, Postpartum anaemia

\section{INTRODUCTION}

Iron deficiency is the most common cause of anaemia worldwide and is most often due to blood loss or chronic dietary insufficiency. ${ }^{1}$ Women who are of reproductive age are particularly at risk owing to blood loss or increased iron demand attributed to menstruation, pregnancy, and lactation. ${ }^{2}$ Postpartum anaemia is a persistent and serious problem globally and especially for being frequent in developing countries, despite prophylactic/therapeutic recommendations by the World Health Organization (WHO). Due to the lack of a globally acceptable definition of postpartum anaemia, a haemoglobin less than $10 \mathrm{gm} \%$ as defined by WHO is used in most studies. Based on this definition the prevalence of postpartum anaemia has been observed to be between 50 to $80 \%$ in developing countries. ${ }^{3}$ Postpartum anaemia is associated with an impaired 
quality of life, reduced cognitive abilities, emotional instability, and depression and constitutes a significant health problem in women of reproductive age. ${ }^{3}$

Iron carbohydrate complexes have revolutionized the treatment of iron deficiency anaemia. Iron carbohydrate complexes should be considered when oral iron is ineffective and for patients who are unable to absorb sufficient iron in the gastrointestinal tract or for whom blood transfusions should be avoided. Injection iron sucrose is one of the commonly used parenteral iron used in postpartum anaemia. Injection iron sucrose is effective but because of the requirement of multiple doses and long stay in hospital, the patients often do not receive the entire iron requirement. Ferric carboxymaltose is a fairly novel iron complex that consists of a ferric hydroxide core stabilized by a carbohydrate shell, allows for controlled delivery of iron to target tissues. Administered intravenously, it is effective in the treatment of irondeficiency anaemia, delivering a replenishment dose of up to $1000 \mathrm{mg}$ of iron during a minimum administration time of around 15 minutes. ${ }^{4}$

Though there are many studies conducted of ferric carboxymaltose in postpartum anaemia, there are very few studies where ferric carboxymaltose is compared with iron sucrose in a head to head study. The current study is conducted to compare the safety and efficacy of ferric carboxymaltose and iron sucrose in women with postpartum anaemia attending to a tertiary hospital in southern part of India.

\section{METHODS}

Current study was conducted in Department of Obstetrics and Gynaecology, Vydehi Institute of Medical Sciences (VIMS) between December 2015 and June 2017. The study protocol was approved by the ethical committee board at the study institution. A written informed consent was received from all patients in the study.

The study was designed as a randomized, single centre, open label, active controlled trial.

\section{Inclusion criteria}

- Women admitted to the institution for delivery and were diagnosed to have postpartum anaemia were considered for screening for inclusion in the study.

- Eligible patients were those aged above 18 years, were diagnosed with postpartum anaemia within 6 weeks of delivery, either vaginal or Caesarean.

- Patient should have had haemoglobin levels between 7 to $9 \mathrm{gm} \%$.

\section{Exclusion criteria}

- Patients were excluded from the study if they were experiencing anaemia with any cause other than iron deficiency anaemia like haemolytic anaemia or thalassemia.

- Anaemia of chronic disease like due to chronic infection was also excluded.

- Patients with clinically significant liver disease or renal disease were excluded.

Patients were randomized in a 1:1 ratio using a randomization table to receive treatment with either injection ferric carboxymaltose (Group 1) or iron sucrose (Group 2). Investigator and patients were not blinded to study drug allocation.

After recruitment, a detailed medical history was taken, and patient underwent a through general physical examination. Baseline haematological investigations were conducted including complete blood count, blood group, $\mathrm{Rh}$ typing, peripheral blood smear, iron profile and urine routine. Iron profile included serum iron, total iron binding capacity, serum ferritin and serum transferrin. The total iron deficit was calculated for each patient using Ganzoni's formula. ${ }^{5}$

Total iron deficit $(\mathrm{mg})=($ Haemoglobin deficit $) \mathrm{x}$ patient weight $(\mathrm{kg}) \times 2.4+500$

Group 1 received injection ferric carboxymaltose at a maximum dose of $1000 \mathrm{mg} /$ week based on the postnatal haemoglobin and body weight. Group 2 received injection iron sucrose at a dose of $200 \mathrm{mg} /$ day on alternate days till the calculated dose was given. $500 \mathrm{mg}$ of ferric carboxymaltose was administered as an infusion in $100 \mathrm{ml} 0.9 \%$ saline over 15 mins. Iron sucrose was administered as $200 \mathrm{mg}$ in $100 \mathrm{ml}$ of $0.9 \%$ NS over 15 mins. After patient received the complete planned dose to meet the iron deficit, patients were followed up at week 2 and at week 6 from the initiation of treatment. Haemoglobin was measured during the follow up visits. Patients were enquired about the adverse events and were documented accordingly during follow-up visits. Any concomitant medications were documented.

The primary efficacy parameter was the change in haemoglobin from baseline at 2 weeks and 6 weeks. The secondary outcome was proportion of patients in each group attaining a target haemoglobin level defined as $>12$ $\mathrm{gm} / \mathrm{dl}$. Adverse events were summarized descriptively.

\section{Statistical analysis}

A formal hypothesis-based sample size was not calculated. A sample size of 100 patients, with 50 patients in each treatment group was considered for the study. Statistical analysis was performed using SPSS Version 21.0 (SPSS Inc., Chicago, IL, USA). Change in haemoglobin from baseline was analysed using Student's t-test for independent samples (continuous variables). Categorical variables were analysed using Fisher's exact test. Results were considered statistically significant when $p<0.05$. 


\section{RESULTS}

One hundred patients diagnosed with postpartum anaemia were recruited in the study. Fifty were randomized to receive injection ferric carboxymaltose (Group 1) and 50 received injection iron sucrose (Group 2). Forty-four patients in ferric carboxymaltose group and 42 patients in injection iron sucrose group completed the 6 weeks study visit. Six patients in ferric carboxymaltose group and 8 women in iron sucrose were lost to follow-up during the study (Figure 1).

The baseline characteristics were in general similar between the treatment groups. The maternal age at inclusion in the study was $24.6 \pm 3.7$ years in the ferric carboxymaltose group compared to $23.8 \pm 4.0$ in the iron sucrose group. There were higher proportion of primipara in the iron sucrose group compared to ferric carboxymaltose group (60\% versus 34\%). This difference was incidental and considered not to have an impact on the response to ferric carboxymaltose or Iron sucrose. The antenatal haemoglobin and postnatal haemoglobin were similar between the groups. There was a statistically significant difference in mean antenatal haemoglobin between groups but the difference was not clinically relevant for the study objectives $(11.4 \pm 0.9 \mathrm{~g} / \mathrm{dl}$ in ferric carboxymaltose compared to $11.8 \pm 0.9$ in iron sucrose group). Other iron parameters like serum ferritin and serum iron were comparable across treatment groups (Table 1).

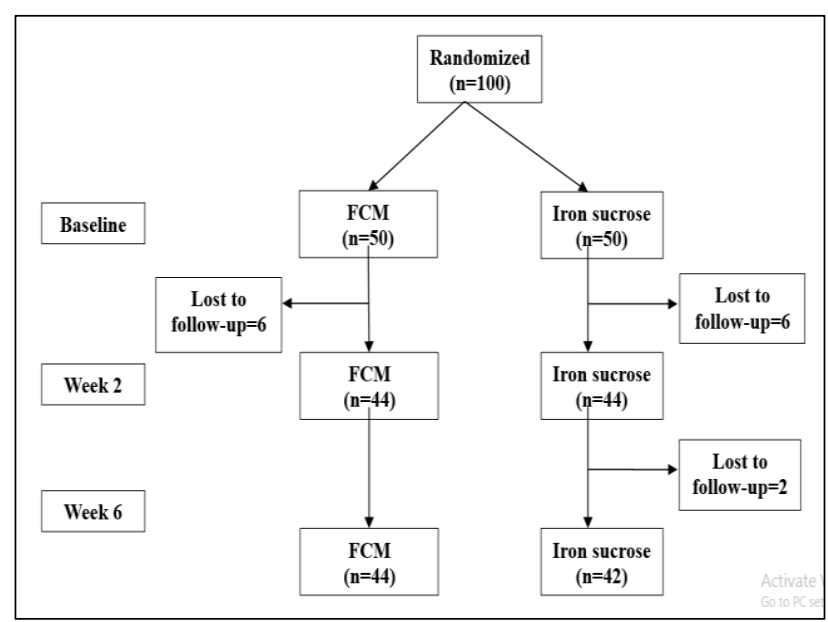

FCM= Ferric carboxymaltose

Figure 1: Subject disposition flow-chart.

Table 1: Baseline characteristics of the group (mean $\pm \mathrm{SD})$.

\begin{tabular}{|c|c|c|c|}
\hline & Group 1 (ferric carboxymaltose) $(\mathrm{n}=50)$ & Group 2 (iron sucrose) $(\mathrm{n}=\mathbf{5 0})$ & $p$ value \\
\hline Maternal age, years & $24.6 \pm 3.7$ & $23.8 \pm 4.0$ & 0.293 \\
\hline Weight, kg & $62.8 \pm 6.1$ & $63.5 \pm 4.7$ & 0.534 \\
\hline Primipara & $17(34.0 \%)$ & $30(60.0 \%)$ & 0.009 \\
\hline Antenal $\mathrm{Hb}, \mathrm{g} / \mathrm{dl}$ (antenatal) & $11.4 \pm 0.9$ & $11.8 \pm 0.9$ & 0.031 \\
\hline Postnatal Hb g/dl (postnatal) & $8.1 \pm 0.5$ & $8.1 \pm 0.5$ & 0.777 \\
\hline Serum ferritin, $\mu \mathrm{g} / \mathrm{L}$ & $13.6 \pm 6.6$ & $14.5 \pm 8.2$ & 0.537 \\
\hline Serum iron, $\mu \mathrm{g} / \mathrm{dL}$ & $42.8 \pm 9.9$ & $47.3 \pm 6.9$ & 0.312 \\
\hline
\end{tabular}

Data are expressed as mean \pm SD or $\mathrm{n}(\%)$.

Table 2: Mean rise in haemoglobin at week 2 and week 6.

\begin{tabular}{|llll|}
\hline & Ferric carboxymaltose $(\mathbf{n}=50)$ & Iron sucrose $(\mathbf{n = 5 0})$ & P-value \\
\hline Mean haemoglobin at week 2, g/dl & $10.71 \pm 0.84$ & $10.30 \pm 0.65$ & 0.010 \\
\hline Mean rise in haemoglobin from baseline at week 2, g/dl & $2.64 \pm 0.91$ & $2.17 \pm 0.76$ & \\
\hline Mean haemoglobin at week 6, g/dl & $12.71 \pm 1.18$ & $12.08 \pm 0.97$ & 0.005 \\
\hline Mean rise in haemoglobin from baseline at week 6, g/dl & $4.65 \pm 1.17$ & $3.96 \pm 1.06$ & 0.005 \\
\hline
\end{tabular}

Data are expressed in mean \pm SD.

At week 2 after the infusion there was an increase in haemoglobin compared to baseline (postnatal) in both groups. The mean increase in haemoglobin from baseline was significantly higher in Ferric carboxymaltose group compared to Iron sucrose group. $(2.64 \pm 0.91$ versus $2.17 \pm 0.76 ; \mathrm{p}=0.010$ ). At Week 6 , the haemoglobin further increased compared to Week 2 in both groups. The mean increase in haemoglobin from baseline was significantly higher in Ferric carboxymaltose group compared to Iron sucrose group $(4.65 \pm 1.17$ versus
$3.96 \pm 1.06 ; \mathrm{p}=0.005$ ) (Table 2). The mean rise in haemoglobin for both study groups is shown graphically in Figure 2.

The target haemoglobin for therapy was $12 \mathrm{~g} / \mathrm{dl}$. At the end of follow up period at 6 weeks, around 77.3\% (34 of 44 ) of patients in the Ferric carboxymaltose group and $50.0 \%$ (21 of 42 ) of patients in iron sucrose achieved the target haemoglobin. This difference was statistically significant $(\mathrm{p}=0.013)$. 
Majority of the patients did not report any adverse events during the study. Around 10\% patients in the Ferric carboxymaltose group and $6 \%$ in the iron sucrose group reported at least one adverse event. In the iron sucrose group fever and myalgia were the most common adverse events reported by 2 patients each reporting the event. One patient in Ferric carboxymaltose group reported urticaria. In the iron sucrose group fever was the most common adverse event with 2 patients reporting it. Apart from that 1 patient reported urinary tract infection. All adverse events were treated promptly, and patients recovered without any sequelae.

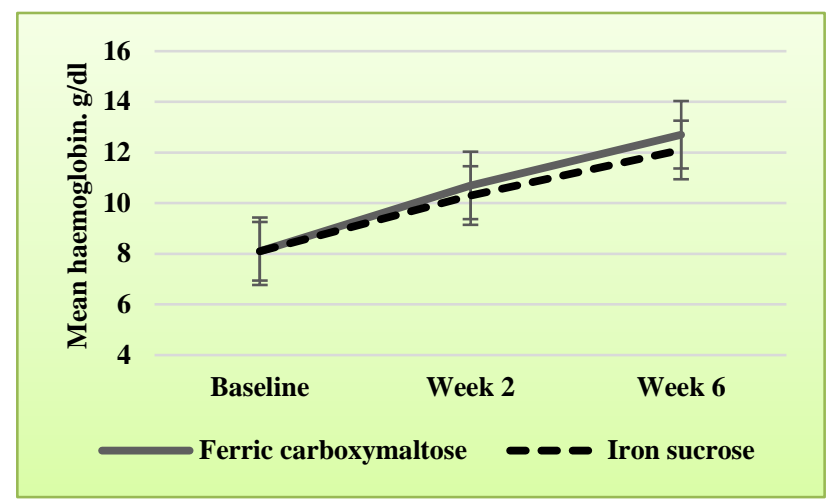

Data expressed as mean $\pm \mathrm{SE}$

Figure 2: Mean haemoglobin at baseline, week 2 and week 6 .

\section{DISCUSSION}

The current study was a single centre randomized control study of injection ferric carboxymaltose and injection iron sucrose in women diagnosed with postpartum anaemia. The key finding of the study is that ferric carboxymaltose demonstrated higher and sustained mean rise in haemoglobin from baseline compared to iron sucrose at both timepoints i.e. week 2 and week 6 in the study. The proportion of patients achieving the target haemoglobin $(12 \mathrm{mg} / \mathrm{dl})$ was also significantly higher in patients receiving ferric carboxymaltose compared to iron sucrose.

Ferric carboxymaltose is compared to iron sucrose earlier for different clinical settings of iron deficiency anaemia. ${ }^{6-}$ 11 The results of present study were similar to the published literature. All these previous studies have shown that injection ferric carboxymaltose increased the mean haemoglobin faster than iron sucrose and higher proportion of patients achieved the target haemoglobin when treated with ferric carboxymaltose compared to iron sucrose. In present study, at week 2, the mean rise in haemoglobin was $2.64 \pm 0.91$ in injection ferric carboxymaltose group compared to $2.17 \pm 0.76$ iron sucrose $(p=0.010)$. Similarly, at week 6 , the mean rise in haemoglobin was $4.65 \pm 1.17$ in injection ferric carboxymaltose group compared to $3.96 \pm 1.06$ iron sucrose $(\mathrm{p}=0.005)$.
In Mahey et al, after 6 weeks, the increase in haemoglobin from baseline was significantly higher in the FCM group than in the ISC group $(4.8 \pm 1.3 \mathrm{~g} / \mathrm{dL}$ versus $3.7 \pm 1.5 \mathrm{~g} / \mathrm{dL} ; \mathrm{P}=0.005) .{ }^{8}$ In Naqash et al, the mean rise in haemoglobin at 4 weeks was relatively higher compared to present results at around $5.49 \mathrm{~g} / \mathrm{dl} .^{6}$ Sharma et al, in a study conducted in patients with postpartum anaemia demonstrated a mean rise in haemoglobin of $3.14 \mathrm{~g} / \mathrm{dl}$ at week 2 in the ferric carboxymaltose group compared to $2.63 \mathrm{~g} / \mathrm{dl}$ in present study at week 2.7 Pfenniger et al also demonstrated numerically higher increase in haemoglobin with ferric carboxymaltose compared to iron sucrose at day 8 but the difference did not meet the statistical significance $(1.83$ versus $0.53 \mathrm{~g} / \mathrm{dl} ; \mathrm{p}=0.1) .^{10}$

In present study, at the end of follow up period at 6 weeks, significantly higher proportion of patients in the ferric carboxymaltose group compared to iron sucrose treatment group achieved the target haemoglobin of 12 gm\% (77.3\% versus 50.0\%). These numbers were similar to Mahey et al where $75 \%$ in the ferric carboxymaltose group and $65 \%$ in the iron sucrose group achieved a similar target haemoglobin. ${ }^{8}$

Some limitations of present study include the smaller sample size and not measuring follow-up iron parameters during the study which would have given good insight on the way study drugs impact the iron stores. Another important limitation of present study is that authors did not follow-up the patients for a longer duration. The superiority of ferric carboxymaltose over iron sucrose may be observed over a short duration as observed in Mahey et al. In the Mahey et al, there was a significantly higher mean rise in haemoglobin in the ferric carboxymaltose group compared to iron sucrose at Week 6 but there was no statistically significant difference was observed between the groups at week $12 .{ }^{8}$ It may indicate that ferric carboxymaltose replenishes irons stores faster and increases haemoglobin earlier compared to iron sucrose, but over a longer duration like 12 weeks both ferric carboxymaltose and iron sucrose may be similar in efficacy as measured by rise in haemoglobin.

Patients tolerated both ferric carboxymaltose and iron sucrose well in present study with no safety concerns identified. Only one patient in ferric carboxymaltose group reported urticaria which was self-limiting.

\section{CONCLUSION}

To conclude, ferric carboxymaltose increased the haemoglobin faster than iron sucrose and significantly higher proportion of patients achieved target haemoglobin within 6 weeks of follow-up. Most patients tolerated ferric carboxymaltose and iron sucrose well. Ferric carboxymaltose may be considered in women with postpartum anaemia for faster rise in haemoglobin while requiring fewer injections compared to iron sucrose. 
Funding: No funding sources

Conflict of interest: None declared

Ethical approval: The study was approved by the Institutional Review Board, Vydehi Institute of Medical Sciences and Research Centre, Bengaluru, India

\section{REFERENCES}

1. Kassebaum NJ, Jasrasaria R, Naghavi M, Wulf SK, Johns N, Lozano R, et al. A systematic analysis of global anaemia burden from 1990 to 2010. Blood. 2014;123(5):615-24.

2. Friedman AJ, Chen Z, Ford P, Johnson CA, Lopez $\mathrm{AM}$, Shander A, et al. Iron deficiency anaemia in women across the life span. J Womens Health (Larchmt). 2012;21(12):1282-9.

3. Milman N. Postpartum anaemia I: definition, prevalence, causes, and consequences. Ann Hematol. 2011;90(11):1247-53.

4. Lyseng-Williamson KA, Keating GM. Ferric carboxymaltose: a review of its use in irondeficiency anaemia. Drugs. 2009;69(6):739-56.

5. Ganzoni AM. Intravenous iron-dextran: therapeutic and experimental possibilities. Schweiz Med Wochenschr. 1970;100(7):301-3.

6. Naqash A, Ara R, Bader GN. Effectiveness and safety of ferric carboxymaltose compared to iron sucrose in women with iron deficiency anaemia: phase IV clinical trials. BMC Womens Health. 2018;18(1):6.

7. Sharma N, Thiek JL, Natung T, Ahanthem SS. Comparative study of efficacy and safety of ferric carboxymaltose versus iron sucrose in post-partum anaemia. J Obstet Gynaecol India. 2017;67(4):253-7.

8. Mahey R, Kriplani A, Mogili KD, Bhatla N, Kachhawa G, Saxena R. Randomized controlled trial comparing ferric carboxymaltose and iron sucrose for treatment of iron deficiency anaemia due to abnormal uterine bleeding. Int $\mathbf{J}$ Gynaecol Obstet. 2016;133(1):43-8.

9. Christoph P, Schuller C, Studer H, Irion O, De Tejada BM, Surbek D. Intravenous iron treatment in pregnancy: comparison of high-dose ferric carboxymaltose vs. iron sucrose. J Perinat Med. 2012;40(5):469-74.

10. Pfenniger A, Schuller C, Christoph P, Surbek D. Safety and efficacy of high-dose intravenous iron carboxymaltose vs iron sucrose for treatment of postpartum anaemia. J Perinat Med. 2012;40(4):397402.

11. Bisbe E, García-Erce JA, Díez-Lobo AI, Muñoz M. Anaemia Working Group España. A multicentre comparative study on the efficacy of intravenous ferric carboxymaltose and iron sucrose for correcting preoperative anaemia in patients undergoing major elective surgery. Br J Anaesth. 2011;107(3):477-8.

Cite this article as: Sheela HS, Neelakanthi A, Nikitha CP. Randomized clinical trial to evaluate ferric carboxymaltose and iron sucrose for treatment of postpartum anaemia in a tertiary hospital. Int $\mathbf{J}$ Reprod Contracept Obstet Gynecol 2018;7:4551-5. 